\title{
Contrasting Effects of WIN 55212-2 on Motility of the Rat Bladder and Uterus
}

\author{
Natalia Dmitrieva and Karen J. Berkley \\ Program in Neuroscience, Florida State University, Tallahassee, Florida 32306-1270
}

Both the uterus and bladder contain cannabinoid $(\mathrm{CB})$ receptors whose functions are poorly understood. Here, in urethaneanesthetized female rats in metestrus, we simultaneously compared the effects of close-arterial injections of the cannabinoid receptor agonist WIN 55,212-2 (WIN2) on uterine contractions (amplitude and rate) and micturition thresholds (MT) assessed by cystometry. Five doses of WIN2 were delivered $(0.01,0.1$, $0.5,1$, and $1.5 \mu \mathrm{mol} / \mathrm{kg}$ ) in three groups: (1) controls; (2) after bladder inflammation with intravesicular turpentine; and (3) after bilateral hypogastric neurectomy (HYPX). In some rats, drugs were delivered via the tail vein. Regarding bladder, WIN2 dose-dependently reduced MTs in all groups. Both bladder inflammation and HYPX significantly increased this effect. Regarding uterus, WIN2 dose-dependently increased uterine contraction amplitude. Bladder inflammation or HYPX significantly decreased this effect. Coinjection of the CB1 antagonist
SR141716A (SR) $(1.5 \mu \mathrm{mol} / \mathrm{kg})$ and WIN2 $(0.5 \mu \mathrm{mol} / \mathrm{kg})$ abolished or reduced the effects of WIN2 in both organs. SR alone had significant effects only after HYPX, reducing both MT and uterine contraction amplitude. The vehicle (0.4\% DMSO) and inactive enantiomer $S(-)$-WIN 55,212-3 were both ineffective. Close-arterial injections of WIN2 $(0.5 \mu \mathrm{mol} / \mathrm{kg})$ produced significantly larger effects in both organs than tail vein injections. These results indicate that, whereas WIN2 reduces bladder motility, it mainly increases uterine motility, likely via CB1 receptors located in the two organs. The opposing effects of bladder inflammation and HYPX on the potency of WIN in the two organs suggest a neurally mediated viscero-visceral interaction in which bladder inflammation influences uterine CB1 sensitivity, possibly by inhibiting adrenergic input to the uterus.

Key words: cannabinoid; hypogastric nerve; inflammation; pelvic organs; lower urinary tract; reproductive organs
Numerous anecdotal reports indicate that cannabinoids (CBs) affect uterine and bladder motility. Thus, cannabis has been used for more than a century to treat dysmenorrhea and menorrhagia (O’Shaughnassy, 1842; Abel, 1980). Furthermore, multiple sclerosis patients report that cannabis alleviates their nocturia and bladder hyperactivity and pain (Consroe et al., 1997); so does $\Delta^{9}$-tetrahydro-cannabinol ( $\Delta^{9}$-THC) (Martyn et al., 1995). Recently, the CB1 receptor was identified in both rodent bladder (Pertwee and Fernando, 1996; Martin et al., 2000) and uterus (Das et al., 1995). CB1 receptors were also identified in terminals of peripheral sensory afferent and sympathetic fibers (Hohman and Herkenham, 1999; Niederhoffer and Szabo, 1999; Malinowska et al., 2001; Niederhoffer et al., 2001).

Together, these reports suggest involvement of local cannabinoid receptors, particularly $\mathrm{CB} 1$ receptors, in regulating uterine and bladder function. Indeed, the endogenous cannabinoid ligand anandamide and endometrial $\mathrm{CB} 1$ receptors participate in regulating receptivity of the mouse uterus for embryo implantation (Schmid et al., 1997; Paria and Dey, 2000). For the bladder, different cannabinoid receptors may become engaged depending on its state; i.e., healthy versus inflamed. Thus, bladder mast cells, which express the CB2 receptor (Facci et al., 1995), increase dramatically during bladder inflammation (Christmas and Rode,

Received March 29, 2002; revised May 16, 2002; accepted May 20, 2002.

This study was supported by The Fishbein Family IC Research Foundation of the Interstitial Cystitis Association and National Institutes of Health Grant RO1 NS11892. We thank Dr. Elisabet Torres for help with statistics and Xavier Bonilla for help with the figures.

Correspondance should be addressed to Dr. Karen J. Berkley, Program in Neuroscience, Copeland Street, Florida State University, Tallahassee, FL 32306-1270. E-mail: kberkley@psy.fsu.edu.

Copyright (C) 2002 Society for Neuroscience $\quad 0270-6474 / 02 / 227147-07 \$ 15.00 / 0$
1991; Sant and Theoharides, 1994). Furthermore, Jagger et al. (1998a,b) compared effects of systemic delivery of anandamide and palmitoylethanolamide (PEA) before and after bladder inflammation on inflammation-induced bladder hyperreflexia. Because both agents reduced hyperreflexia after inflammation, but only anandamide prevented hyperreflexia before inflammation, they concluded that $\mathrm{CB} 2$ receptors become involved in regulating bladder motility only after inflammation (Jagger et al., 1998a). These results, however, do not necessarily imply direct activation of CB2 receptors, because PEA does not bind directly to them (Showalter et al., 1996; Jonsson et al., 2001).

Here we developed a protocol to characterize the influence of cannabinoid agents simultaneously on bladder and uterine motility. We then used this protocol to examine effects of close-arterial injections of the mixed CB1/CB2 receptor agonist $R(+)$-WIN $55,212-2$ (WIN2) on the frequency and amplitude of uterine contractions and on cystometrically measured micturition thresholds (MTs) before and after bladder inflammation (i.e., on the volume of saline infused into the bladder that induced a bladder contraction). To determine the contribution of $\mathrm{CB} 1$ receptors, we examined effects when WIN2 was delivered together with the CB1-specific antagonist SR141716A (SR). To provide evidence about the site of action of the ligand, we compared responses when the same dose of WIN2 was injected close-arterially or via the tail vein.

Hypogastric nerve activity can inhibit micturition (de Groat and Theobald, 1976) and is involved in controlling uterine activity and nociception (Sato et al., 1989, 1996; Temple et al., 1999; Dmitrieva et al., 2001). Because WIN2 modulates sympathetic activity in rodents (Malinowska et al., 1997; Niederhoffer and Szabo, 1999) and $\Delta^{9}$-THC inhibits noradrenaline release in rat 


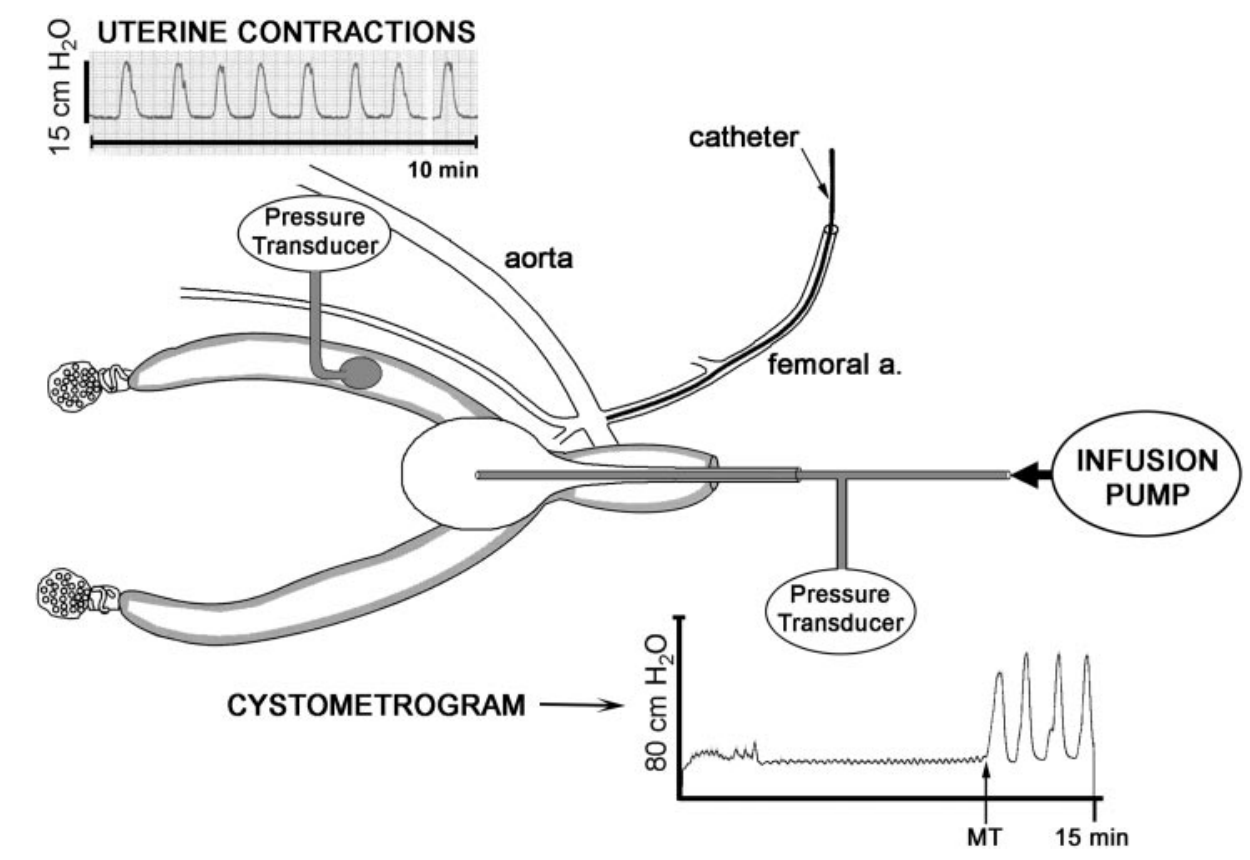

Figure 1. Diagram of the experimental setup and example records of uterine contractions (top) and a cystometrogram (bottom). For details, see Materials and Methods.

isolated atria and vas deferens (Ishac et al., 1996), it is possible that cannabinoids influence bladder and uterine motility via the hypogastric nerve. We tested this hypothesis by studying effects of WIN2 after bilateral hypogastric neurectomy (HYPX).

\section{MATERIALS AND METHODS}

\section{Animal preparation}

Adult female Sprague Dawley rats (220-270 gm) were used. They were housed individually in transparent plastic cages with chip bedding and maintained on a $12 \mathrm{hr}$ light/dark cycle. Before the experiment, their estrous stages were monitored daily via vaginal smears obtained $2 \mathrm{hr}$ after lights on for at least 3 weeks before the experiment. Only rats with at least three regular $4 \mathrm{~d}$ estrous cycles were used. They were in metestrus on the day of the experiment.

Rats were anesthetized with urethane $(1.2 \mathrm{gm} / \mathrm{kg}$, supplemented as needed). Body temperature was maintained at $\sim 37^{\circ} \mathrm{C}$ throughout the experiment by a heating pad and warming lamp. To prepare for closearterial injections, an incision was made in the skin of the inner left hindleg. The femoral artery was freed from the surrounding tissue and separated from the vein. A thin polyethylene catheter (outer diameter of $0.61 \mathrm{~mm}$ ) was inserted deeply into the femoral artery $(2.0-2.5 \mathrm{~mm})$ so that its tip rested near arterial branches to the bladder and left uterine horn. It was then secured in place (Fig. 1). In some rats, the tail vein was catheterized instead of the femoral artery.

To prepare for cystometrograms (CMGs), the bladder was catheterized transurethrally with polyethylene tubing (outer diameter of 0.97 $\mathrm{mm}$ ), with the tip of the catheter placed $\sim 2 \mathrm{~mm}$ within the lumen of the bladder so that the tip did not contact the bladder walls (Fig. 1). A suture was tied around the surrounding skin to prevent leakage.

To prepare for measuring the amplitude and rate of uterine contractions, a ventral midline incision was made to expose the bladder and left uterine horn. In all rats, a small incision was made near the midrostral end of the left uterine horn. A water-filled catheter with a small latex balloon on its end (diameter of $\sim 2 \mathrm{~mm}$ ) was inserted into the lumen so that the balloon, secured by a stitch, rested $\sim 1 \mathrm{~cm}$ above the uterine body. The uterine catheter was connected to another small-volume pressure transducer and amplifier (Fig. 1). The abdomen was covered with a saline-dampened pad. Both the bladder and uterine catheters were then connected to small-volume pressure transducers, whose signals were amplified and relayed to strip chart and videotape recorders for offline analyses.

\section{Cystometrograms}

For bladder motility, MTs were assessed via CMGs, which measured pressure within the bladder while it was slowly filled $(0.05 \mathrm{ml} / \mathrm{min})$ with saline via the transurethral catheter to a maximum volume of $1 \mathrm{ml}$ (to avoid overinflation). MT was defined as either the intraluminal volume that produced the first micturition contraction, or, if no contraction occurred (rarely), $1 \mathrm{ml}$. Figure 1 provides an example CMG record.

\section{Uterine contractions}

The amplitude and frequency of spontaneous uterine contractions were recorded as they passed over the uterine balloon and were measured manually from the strip chart records (Fig. 1). Mean amplitude of uterine contractions was calculated from three to five contractions that were measured between the times that CMGs were being run. Mean rate of uterine contractions was calculated in 5 min intervals during a $25 \mathrm{~min}$ period before and a $30 \mathrm{~min}$ period after administration of each drug.

\section{Bladder inflammation}

After the baseline MT was established, $0.5-0.8 \mathrm{ml}$ of $50 \%$ turpentine oil (in olive oil) was instilled into the bladder. At approximately hourly intervals afterward, the bladder was emptied by gentle pressure and suction, and additional CMGs were performed.

\section{Bilateral hypogastric neurectomy}

In some rats, just before the uterus was catheterized, the left and right hypogastric nerves were identified as they exited caudally from the inferior mesenteric ganglion (IMG). A $1 \mathrm{~cm}$ length of both nerves was freed from surrounding connective tissue. A snare of 4.0 silk suture was loosely tied around each nerve. At the appropriate point in the experiment, each snare could be pulled through the hypogastric nerves to cut them (HYPX). This technique had been used successfully previously to cut the vagus nerve (Hubscher and Berkley, 1995). Baseline MT was also established before HYPX.

\section{Experimental design}

Study 1: dose-response to WIN2. A dose-response function for closearterial injections of WIN2 was obtained using five doses of WIN2 (0.01, $0.1,0.5,1$, and $1.5 \mu \mathrm{mol} / \mathrm{kg}$ ) and compared with the effect of a high dose of the inactive enantiomer $S(-)$-WIN 55,212-3 (WIN3) $(1 \mu \mathrm{mol} / \mathrm{kg})$ and the vehicle $(0.4 \%$ DMSO). The agents were tested in three different groups of rats: (1) control group, rats without either bladder inflammation or HYPX, (2) rats with bladder inflammation, and (3) rats with HYPX. All rats received a close-arterial injection of the vehicle and/or W IN3 before one or two doses of WIN2 were delivered. The drugs were tested $2-5 \mathrm{hr}$ after the onset of inflammation because previous reports have demonstrated that the maximum bladder hyperreflexia develops by $2 \mathrm{hr}$ and is maintained for an additional $3 \mathrm{hr}$ (McMahon and Abel, 1987; Dmitrieva et al., 1997). In the HYPX group, all treatments were per- 


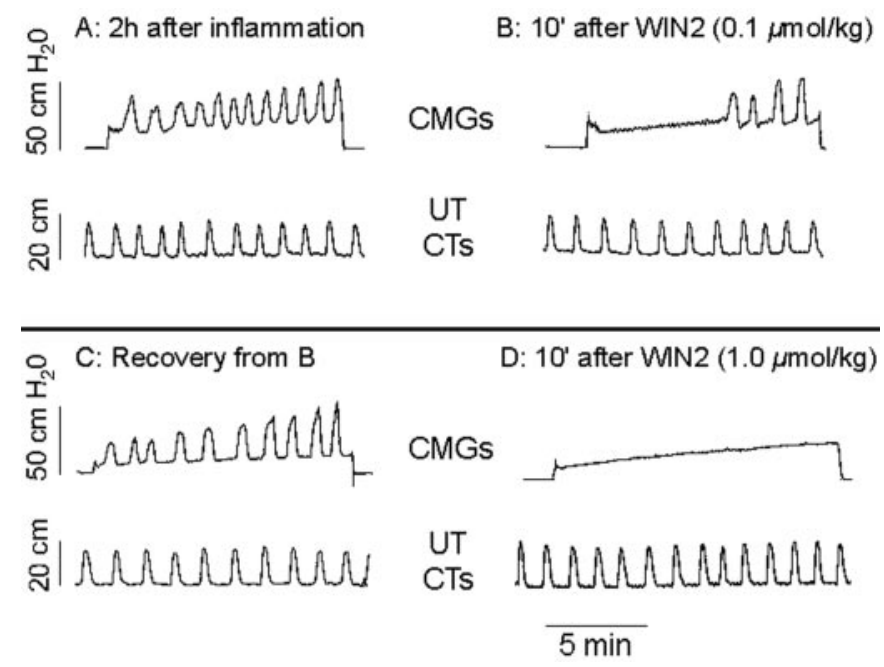

Figure 2. Examples of cystometrograms and uterine contractions recorded in the same rat before and after close-arterial injections of two doses of WIN2. Bladder pressure (top traces in $A-D$ ) and uterine pressure (bottom traces in $A-D$ ) were recorded simultaneously. The bladder of this animal had been inflamed with $50 \%$ turpentine (inflammation). $A$ shows a cystometrogram obtained $3 \mathrm{hr}$ after inflammation when the hyperreflexia was fully developed. The very low micturition threshold is obvious in this record. $B$ shows the next cystometrogram obtained 15 min after 0.1 $\mu \mathrm{mol} / \mathrm{kg}$ WIN2 had been injected through the left femoral artery. Here the micturition threshold was greatly increased compared with $A$. $C$ is another cystometrogram obtained $2 \mathrm{hr}$ later, showing a return of the bladder hyperreflexia to its preinjection level. $D$ shows another cystometrogram obtained 15 min after a higher dose of WIN2 $(1.0 \mu \mathrm{mol} / \mathrm{kg})$ was injected. Here the bladder did not contract during the $15 \mathrm{~min}$ cystometry test, indicating an increase in micturition threshold greater than that after the lower dose of WIN2. Unlike micturition thresholds, uterine contractions in the rat with bladder inflammation were changed very little by either dose of WIN2. UT CTs, Uterine contractions.

formed 2-4 hr after neurectomy because previous studies have shown that MTs are decreased beginning $1.5 \mathrm{hr}$ after HYPX (Dmitrieva and Berkley, 2001).

Study 2: influence of CB1 antagonist. Three groups of rats (control, bladder inflammation, and HYPX) received close-arterial codelivery of WIN2 and SR ( 0.5 and $1.5 \mu \mathrm{mol} / \mathrm{kg}$, respectively). In the other two groups (normal and HYPX), only SR $(1.5 \mu \mathrm{mol} / \mathrm{kg})$ was injected.

Study 3: close-arterial versus tail vein. One group of intact controls (i.e., without either bladder inflammation or HPYX) received one dose of WIN2 $(0.5 \mu \mathrm{mol} / \mathrm{kg})$ delivered via the tail vein.

The general protocol for all three studies was as follows. After surgical preparations, two or three baseline CMGs were performed at $\sim 30 \mathrm{~min}$ intervals before any treatment. Next, either nothing was done (control group) or the bladder was inflamed or HYPX was performed by pulling the snares through the nerves. CMGs were then repeated in control rats, or $2-3 \mathrm{hr}$ after bladder inflammation, or $2 \mathrm{hr}$ after HYPX. The vehicle was then injected. Ten minutes after vehicle injection, another CMG was obtained. After "recovery" from the vehicle $(<20 \mathrm{~min}$, effects were negligible), another agent (WIN3, or one dose of WIN2 or WIN2 plus SR or SR alone) was delivered. Approximately $10 \mathrm{~min}$ later, another CMG was obtained. After recovery from the first dose of WIN2, CMGs were repeated at $1 \mathrm{hr}$ intervals until recovery occurred $(1.5-2 \mathrm{hr}$; see Results). Then another dose of WIN2 was delivered, and the process was sometimes repeated once more. An example of such a protocol for two doses of WIN2 is shown in Figure 2. For any one subject, at most, three doses of WIN2 in addition to the vehicle control were tested in haphazard order.

\section{Data analysis and statistics}

The mean change in MTs and uterine contraction amplitude before and after drug administrations and the time at which full recovery from the previous dose occurred were tested as appropriate by Student's $t$ test or one- or two-way repeated-measures ANOVAs, followed by Dunnett's

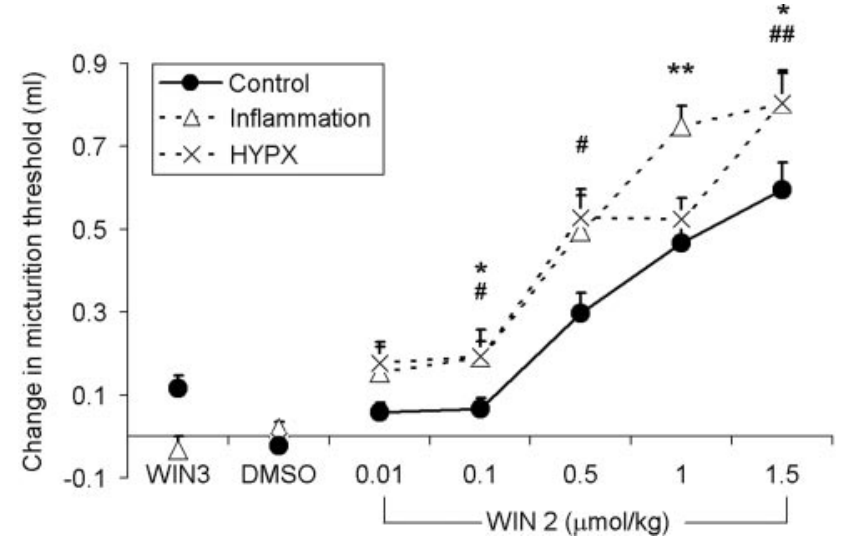

Figure 3. Effect of WIN2 on micturition thresholds in rats with bladders inflamed with $50 \%$ of turpentine (Inflammation), bilateral hypogastric neurectomy (HYPX), and no manipulation (Control). Micturition thresholds were measured before and $15 \mathrm{~min}$ after injection of DMSO, 1.0 $\mu \mathrm{mol} / \mathrm{kg}$ WIN3, or five doses of WIN2. In all groups, WIN2 increased micturition thresholds in a dose-dependent manner $(p<0.005)$. However, the dose-response functions for the inflamed and HYPX rats were shifted to the left relative to control rats. Significant differences between bladder inflamed and control rats for different doses of WIN2: ${ }^{*} p<0.05$; ${ }^{* *} p<0.001$. Significant differences between HYPX and control rats: $\# p<0.05 ; \# \# p<0.005$.

post hoc analysis. Changes in the rate of uterine contractions were analyzed using the Friedman test, followed by Wilcoxon post hoc tests. Significance levels were set at $p<0.05$.

\section{Drugs}

The active cannabinoid receptor agonist WIN2 and the inactive enantiomer WIN3 were purchased from Sigma (St. Louis, MO). The specific CB1 receptor antagonist SR was a gracious gift from the National Institute of Drug Abuse (Bethesda, MD). Drugs were diluted with saline from a stock solution of $50 \mathrm{~mm}$ dissolved in $100 \%$ DMSO. All drugs were delivered by slowly injecting them in a volume of $0.1-0.15 \mathrm{ml}$ and then flushing the drug thorough with $0.1 \mathrm{ml}$ of heparinized saline.

\section{RESULTS}

\section{Effects of bladder inflammation and HYPX on bladder and uterine motility}

As expected from numerous previous studies (McMahon and Abel, 1987; Dmitrieva et al., 1997), bladder inflammation significantly decreased MTs (Fig. 3, compare the first CMG record with a baseline CMG that is shown in Fig. 1). The mean decrease here was $0.38 \pm 0.05 \mathrm{ml}$ (mean $\pm \mathrm{SE} ; p<0.001$ ). As also expected, HYPX significantly decreased MTs but to a lesser extent: $0.2 \pm 0.06 \mathrm{ml}$ (mean $\pm \mathrm{SE} ; p<0.05$ ). Unexpectedly, bladder inflammation also significantly affected uterine motility by decreasing uterine contraction rate. HYPX, on the other hand, significantly affected uterine motility mainly by increasing contraction amplitude. These latter two findings have been reported in full previously (Dmitrieva et al., 2001).

\section{Effects of WIN2 on bladder motility}

As shown in Figure 3, WIN2 significantly increased MTs in a dose-response manner in all groups. The effects after bladder inflammation and HYPX did not differ significantly from each other, but both were significantly greater than in the intact, uninflamed condition $(p<0.05)$. Note that neither DMSO alone nor WIN3 $(1 \mu \mathrm{mol} / \mathrm{kg})$ produced effects on MT in any condition. Examples of some of the effects of WIN2 on MTs are shown in Figure 2.

In most rats, WIN2 had been injected more than once in 


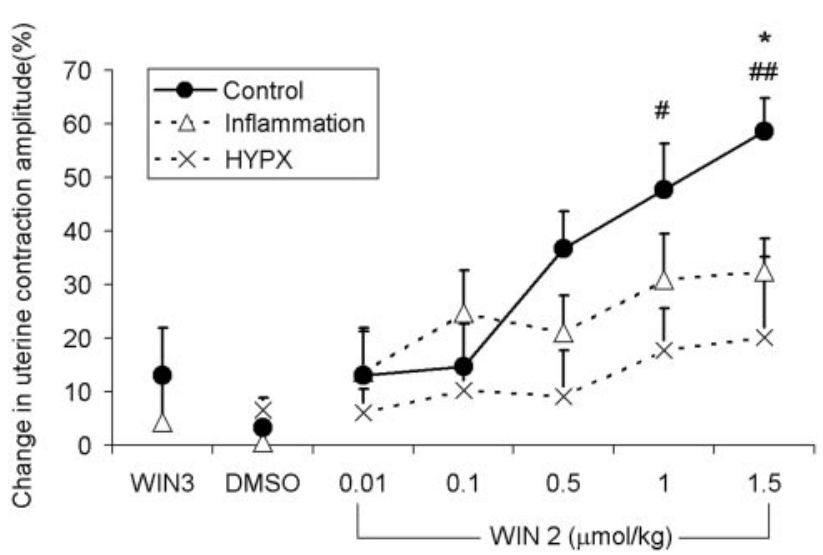

Figure 4. Effect of WIN2 on the amplitude of uterine contractions in the same rats as shown in Figure 3. In control rats and in rats with bladder inflammation, WIN2 increased micturition thresholds in a dosedependent manner (control, $p<0.001$; inflammation, $p<0.005$; however, HYPX, $p>0.3$ ). In contrast with the bladder, the effects of WIN2 in rats after bladder inflammation or HYPX were decreased or abolished, respectively. Significant differences between bladder-inflamed and control rats for different doses of WIN2: ${ }^{*} p<0.05$. Significant differences between HYPX and control rats: $\# p<0.05$; \# $p<0.001$.

different doses. When this procedure was followed, WIN2 was administered only after the MT from the previous dose had returned to the preinjection value (Fig. 2). This recovery period in all cases was $\sim 1.5-2 \mathrm{hr}$ (confirmed by ANOVA; $p>1.0$ ).

\section{Effects of WIN2 on uterine motility}

To determine whether repetitive CMGs might influence the response of the uterus to WIN2, the bladder of three to four rats in each group was catheterized, but $\mathrm{CMGs}$ were not done. The responses of the uterus to WIN2 in these two groups did not differ (results not shown); therefore, the data from both groups was combined. The combined results are presented in Figure 4.

In contrast to the reduction of bladder motility by WIN2 (i.e., increased MTs), WIN2 significantly increased uterine motility. Figure 4 shows that WIN2 increased the amplitude of uterine contractions in a dose-dependent manner $(p<0.001)$ in control rats, as well as in rats with bladder inflammation $(p<0.005)$ but not in rats with HYPX $(p>0.3)$. The latency of this effect was $<1 \mathrm{~min}$. However, again in contrast with the bladder, the effect of WIN2 on uterine contraction amplitude was reduced after bladder inflammation and nearly abolished after HYPX. Thus, the effect of $1.5 \mu \mathrm{mol} / \mathrm{kg}$ WIN2 in the rats with an inflamed bladder was significantly smaller than in control rats $(p<0.05)$. In HYPX rats, the response to 1 and $1.5 \mu \mathrm{mol} / \mathrm{kg}$ WIN2 was significantly lower compared with that observed in control rats $(p<0.05$ and 0.01 for 1 and $1.5 \mu \mathrm{mol} / \mathrm{kg}$, respectively).

WIN2 influenced the rate of uterine contractions to a lesser extent than it did the amplitude of uterine contractions (results not shown). The two highest doses decreased the rate only briefly $(\sim 20 \mathrm{~min})$ in control rats, as well as a middle dose $(0.5 \mu \mathrm{mol} / \mathrm{kg})$ in HYPX rats $(p<0.05)$. There were no significant effects in rats with bladder inflammation.

DMSO and WIN3 failed to produce significant effects on uterine motility in any condition.

\section{Effects of the CB1 antagonist SR141716A}

As shown in Figure $5 A$, injections of SR plus WIN2 prevented reductions by WIN of MTs in all groups $(p<0.005$, significant difference between the effect of SR plus WIN2 and WIN2 alone).
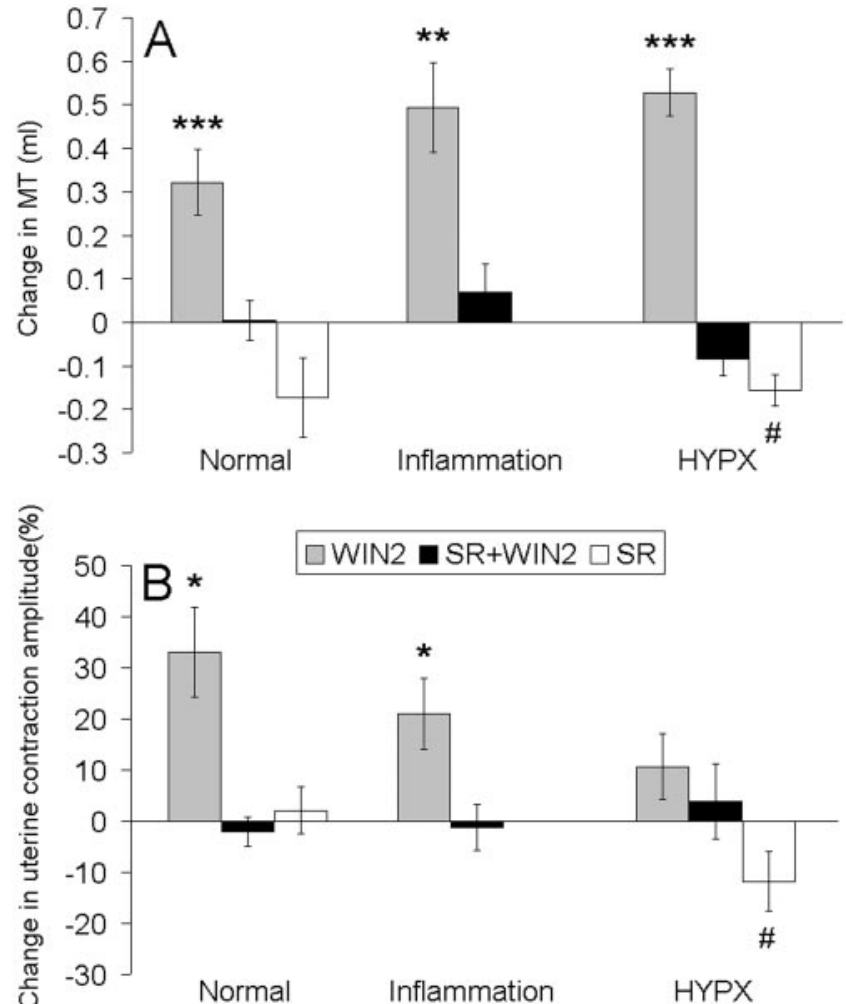

Figure 5. Effect on micturition thresholds $(A)$ and uterine contraction amplitudes $(B)$ of the coadministration of the CB1 antagonist SR and WIN2 (SR+WIN2) compared with the administration of WIN2 alone (WIN2) and SR alone (SR). The dose of SR (alone or in combination with WIN2) was $1.5 \mu \mathrm{mol} / \mathrm{kg}$. The dose of WIN2 (alone or in combination with SR) was $0.5 \mu \mathrm{mol} / \mathrm{kg}$. Significant difference between WIN2 and SR plus WIN2: $* p<0.05 ; * * p<0.01 ; * * * p<0.005$. Significant difference between WIN2 and SR: \#p $<0.05$.

As shown in Figure 5B, SR plus WIN2 also prevented increases by WIN2 of uterine contraction amplitude in control rats and those with bladder inflammation $(p<0.05)$ but not in rats with HYPX.

Control rats and those with HYPX were also treated with 1.5 $\mu \mathrm{mol} / \mathrm{kg}$ SR alone. Both MTs and uterine contraction amplitudes were significantly reduced with HYPX $(p<0.05)$ (Fig. $5 A, B)$ but were unchanged in control rats.

\section{Close-arterial versus tail vein injections}

As shown in Figure 6, the effects of WIN2 on both the bladder and uterus were significantly greater when injected via the closearterial route than via the tail vein $(p<0.05)$.

\section{DISCUSSION}

Using a procedure that permits simultaneous observation of bladder and uterine motility, we found that, whereas WIN2 dosedependently decreased bladder motility, it dose-dependently increased uterine motility in healthy control rats. Whereas both bladder inflammation and HYPX increased the effects of WIN2 on the bladder, the two manipulations decreased the effects of WIN2 on the uterus. These results indicate that cannabinoids exert opposing actions on the bladder and uterus.

When the CB1 receptor antagonist SR was coinjected with WIN2, the effects of WIN2 in both organs were reduced or abolished, suggesting that at least some of those effects occurred by way of CB1 receptors in both organs. WIN2 was more effective when injected close to the organs than when injected via a more 

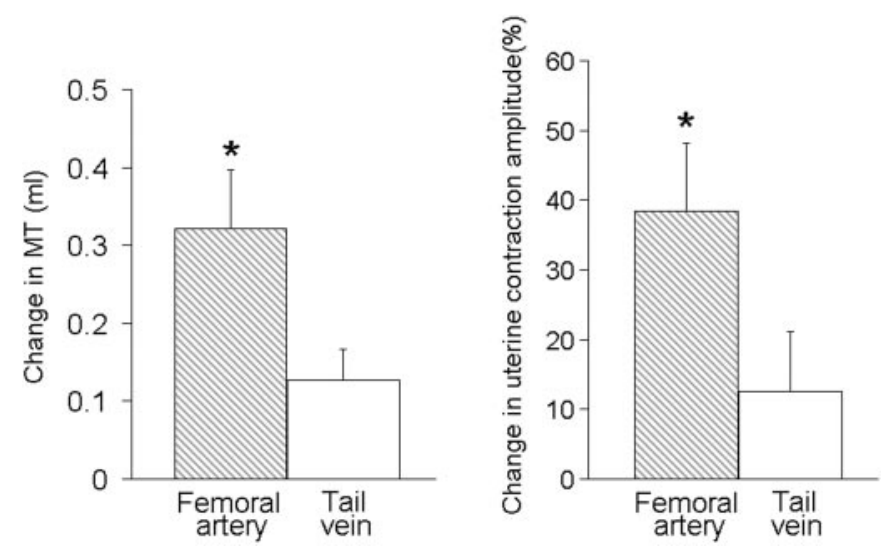

Figure 6. Comparison of the effect of $0.5 \mu \mathrm{mol} / \mathrm{kg}$ W IN2 on micturition thresholds $(A)$ and amplitude of uterine contractions $(B)$ when injected close-arterially versus via the tail vein. ${ }^{*} p<0.05$.

circuitous systemic route through the tail vein, supporting the conclusion that some of the effects occurred locally within the organs.

\section{Effects of cannabinoids on bladder motility}

The results here on the bladder support previous studies demonstrating the existence of CB1 receptors in the bladder, which, when activated, reduce bladder motility (Pertwee and Fernando, 1996; Martin et al., 2000). The fact that, in the present study, bladder inflammation increased the potency of WIN2 suggests an upregulation of CB1 receptors in the bladder in this condition. This suggestion is supported by recent findings that CB1 receptors are upregulated in the mouse intestine after intestinal inflammation (Izzo et al., 2001).

Previous studies have suggested that CB2 receptors become involved in bladder hyperreflexia after bladder inflammation (Jagger et al., 1998b) and that both CB1 and CB2 receptors are involved in the referred hyperalgesia that is produced by bladder inflammation (Farquhar-Smith and Rice, 2001). What was somewhat surprising here, therefore, is that the effects of WIN2, which is a mixed CB1/CB2 agonist (Pertwee, 1999), were completely, rather than only partially, reversed by SR. Although this result suggests that, even in the inflamed bladder, all of the effects of WIN2 may have occurred by way of CB1 receptors, definitive support for this suggestion requires demonstrating that closearterial injections of a CB2 antagonist do not alter the effects of WIN2. Furthermore, it is possible that WIN2 acts on CB2 receptors at higher doses than the middle dose tested here, but that seems unlikely, because, although WIN2 is a CB1 and CB2 receptor antagonist, it actually exhibits a "marginal selectivity for CB2 receptors" (Pertwee, 1999).

The fact that the same dose of WIN2 was more effective in reducing MT when delivered by close-arterial injection than by tail vein injection suggests that the effects were occurring locally within the bladder, possibly on peripheral afferent receptors or sympathetic terminal fibers (Ishac et al., 1996; Richardson et al., 1998; Malinowska et al., 2001). It is likely that the effects were not entirely local, however, because CB1 receptors have been identified or shown to act in rat brainstem sites in the vicinity of regions that control bladder function, such as periaqueductal gray, pons, hypothalamus, and basal ganglia (Matsuda et al., 1993; Fowler, 1998; Pettit et al., 1998; Athwal et al., 2001; Palazzo et al., 2001), as well as in lumbar spinal cord (Hohmann et al., 1999; Drew et al., 2000).
The mechanism by which HYPX increased the potency of WIN2 on the bladder likely differs from that produced by bladder inflammation. Indeed, this increased potency was surprising, given reports that sympathetic terminals might contain CB1 receptors (Malinowska et al., 2001). The receptors in that study, however, supplied the heart, and the same situation may not apply to the sympathetic supply of the bladder. Studies on heteroreceptor interactions in guinea pig hippocampus slices suggest that cannabinoid receptors may influence tonic inhibition from $\alpha 2$ adrenoreceptors (Schlicker et al., 1997; Schlicker and Göthert, 1998). If this type of interaction is the case within the bladder, increased sensitivity to WIN2 in denervated bladder may be attributable to a disinhibition of local CB1 receptors, possibly located on peripheral pelvic nerve afferents like they are in other lumbar spinal afferents (Hohmann and Herkenham, 1999; Ahluwalia et al., 2000).

\section{Effects of cannabinoids on uterine motility}

In intact, uninflamed control rats, WIN2 increased the amplitude of uterine contractions with only minor effects on the rate of contractions at the highest doses. The effects were eliminated by coadministration of SR. These results support previous studies demonstrating the expression of $\mathrm{CB} 1$ receptors in the rodent uterus (Das et al., 1995).

However, whereas coadministration of SR and WIN2 eliminated the effect of WIN2 on the uterus in control rats and rats with an inflamed bladder, it failed to do so in HYPX rats. Furthermore, treatment with SR alone significantly reduced uterine contraction amplitude. These findings suggest that functional $\mathrm{CB} 1$ receptors may still be present in the neurectomized uterus, supporting a postsynaptic action of WIN2 in the uterus. One such postsynaptic mechanism could be an interaction between CB1 and postsynaptic adrenergic receptor signal-transduction mechanisms in the uterus. The $\alpha$-adrenergic receptor is a likely candidate for this role because its activation has been found to increase uterine motility (Ibuki et al., 1997). Cocaine also has been found to exert a stimulatory effect in the uterus, acutely increasing the contraction duration of human myometrial strips (Monga et al., 1993). The mechanism of cocaine action in the human uterus has been suggested to be an inhibition of cAMP production induced by activating adrenoreceptors (Wang et al., 1996). The cAMP is a converging point for signal-transduction pathways activated by different modulators of uterine contractility (Okamura et al., 2000). Reports showing that cannabinoid agonists such as $\Delta^{9}$ THC and anandamide inhibited the forskolin-stimulated cAMP formation in endometrium through activation of the CB1 receptor coupled to G-proteins (Das et al., 1995) support the possibility that, like cocaine, WIN2 may inhibit noradrenaline-induced accumulation of cAMP. Therefore, a possible interaction between sympathetic neurotransmitters and cannabinoid receptors may occur at the level of cAMP production.

It is also possible that cannabinoids regulate uterine contractility indirectly by modulating production of prostaglandins (PGEs). THC has been shown to elevate PGE levels in uterus venous blood (Jordan and Castracane, 1976). Prostaglandins such as $\mathrm{PGE}_{2}$ and $\mathrm{PGF}_{2 \alpha}$ have been found to increase the force of spontaneous contractions (Krall et al., 1984). The action may also lead to a downregulation of cAMP synthesis because $\mathrm{PGE}_{2}$ and $\mathrm{PGF}_{2 \alpha}$ have been found to inhibit cAMP accumulation in rat myometrium (Krall et al., 1984; Goureau et al., 1990). 


\section{Interaction between bladder and uterus}

The potency of WIN2 on uterine motility was reduced in rats with bladder inflammation. One possible mechanism for this viscero-visceral interaction is that bladder inflammation influences the sensitivity of uterine CB1 receptors by way of the hypogastric nerves. In support of such neurogenic control of uterine motility from the bladder is that HYPX both significantly increases the amplitude of spontaneous uterine contractions (Dmitrieva et al., 2001) and, as shown here, nearly abolishes the ability of WIN2 to increase contraction amplitude. One possible scenario is that hypogastric afferents from the bladder that have been activated by inflammation (Moss et al., 1997) convey that information to the T13-L3 segments of the spinal cord (Vera and Nadelhaft, 1992), thereby inhibiting the output of presynaptic neurons that synapse on postganglionic neurons in the IMG whose fibers terminate in the uterus (Papka et al., 1996) and, as suggested above, enhance uterine contractility. HYPX would eliminate this route.

The fact that bladder inflammation decreased the potency of the exogenous cannabinoid ligand WIN2 in the uterus suggests that actions of endogenous cannabinoids, especially anandamide, which is abundant in the uterus (Das et al., 1995), might also be influenced by bladder inflammation. Such a situation would compromise healthy uterine functions. A similar situation might exist for other visceral organs because cannabinoid receptors are expressed in many of them (Joy et al., 1999). Furthermore, this modulation might contribute to the phenomenon of "viscerovisceral-referred hyperalgesia" in which pathophysiology in one visceral domain is found to increase pain associated with pathophysiology in another visceral domain (Giamberardino et al., 1997, 2001, 2002; Berkley et al., 2001), as well as to clinical observations demonstrating the co-occurrence of interstitial cystitis, dysmenorrhea, irritable bowel syndrome, and fibromyaglia (Alagiri et al., 1997).

\section{REFERENCES}

Abel EL (1980) Marijuana: the first twelve thousand years. New York: Plenum.

Ahluwalia J, Urban L, Capogna M, Bevan S, Nagy I (2000) Cannabinoid 1 receptors are expressed in nociceptive primary sensory neurons. Neuroscience 100:685-688.

Alagiri M, Chottiner S, Ratner V, Slade D, Hanno PM (1997) Interstitial cystitis: unexplained associations with other chronic disease and pain syndromes. Urology 49:52-57.

Athwal BS, Berkley KJ, Hussain I, Brennan A, Craggs M, Sakakibara R, Frackowiak RS, Fowler CJ (2001) Brain responses to changes in bladder volume and urge to void in healthy men. Brain 124:369-377.

Berkley KJ, Cason A, Jacobs H, Bradshaw H, Wood E (2001) Vaginal hyperalgesia in a rat model of endometriosis. Neurosci Lett 306:185-188.

Christmas TJ, Rode J (1991) Characteristics of mast cells in normal bladder, bacterial cystitis and interstitial cystitis. Br J Urol 68:473-478.

Consroe P, Musty R, Rein J, Tillery W, Pertwee R (1997) The perceived effects of smoked cannabis on patients with multiple sclerosis. Eur Neurol 38:44-48.

Das SK, Paria BC, Chakraborty I, Dey SK (1995) Cannabinoid ligandreceptor signaling in the mouse uterus. Proc Natl Acad Sci USA 92:4332-4336.

de Groat WC, Theobald RJ (1976) Reflex activation of sympathetic pathways to vesical smooth muscle and parasympathetic ganglia by electrical stimulation of vesical afferents. J Physiol (Lond) 259:223-237.

Dmitrieva N, Shelton D, Rice AS, McMahon SB (1997) The role of nerve growth factor in a model of visceral inflammation. Neuroscience 78:449-459.

Dmitrieva N, Johnson OL, Berkley KJ (2001) Bladder inflammation and hypogastric neurectomy influence uterine motility in the rat. Neurosci Lett 313:49-52.

Drew LJ, Harris J, Millns PJ, Kendall DA, Chapman V (2000) Activation of spinal cannabinoid 1 receptors inhibits C-fibre driven hyperexcitable neuronal responses and increases $\left[{ }^{35} \mathrm{~S}\right] \mathrm{GTP}$ gammaS binding in the dorsal horn of the spinal cord of noninflamed and inflamed rats. Eur J Neurosci 12:2079-2086.
Facci L, Dal Toso R, Romanello S, Buriani A, Skaper SD, Leon A (1995) Mast cells express a peripheral cannabinoid receptor with differential sensitivity to anandamide and palmitoylethanolamide. Proc Natl Acad Sci USA 92:3376-3380.

Farquhar-Smith WP, Rice AS (2001) Administration of endocannabinoids prevents a referred hyperalgesia associated with inflammation of the urinary bladder. Anesthesiology 94:507-513.

Fowler CJ (1998) Brain activation during micturition. Brain 121:2031-2032.

Giamberardino MA, Berkley KJ, Iezzi S, de Bigontina P, Vecchiet L (1997) Pain threshold variations in somatic wall tissues as a function of menstrual cycle, segmental site and tissue depth in non-dysmenorrheic women, dysmenorrheic women and men. Pain 71:187-197.

Giamberardino MA, De Laurentis S, Affaitati G, Lerza R, Lapenna D, Vecchiet L (2001) Modulation of pain and hyperalgesia from the urinary tract by algogenic conditions of the reproductive organs in women. Neurosci Lett 304:61-64.

Giamberardino MA, Berkley KJ, Affaitati G, Lerza R, Centurione L, Lapenna D, Vecchiet L (2002) Influence of endometriosis on pain behaviors and muscle hyperalgesia induced by a ureteral calculosis in female rats. Pain 95:247-257.

Goureau O, Tanfin Z, Harbon S (1990) Prostaglandins and muscarinic agonists induce cyclic AMP attenuation by two distinct mechanisms in the pregnant-rat myometrium. Interaction between cyclic AMP and $\mathrm{Ca}^{2+}$ signals. Biochem J 271:667-673.

Hohmann AG, Herkenham M (1999) Localization of central cannabinoid CB1 receptor messenger RNA in neuronal subpopulations of rat dorsal root ganglia: a double-label in situ hybridization study. Neuroscience 90:923-931.

Hohmann AG, Briley EM, Herkenham M (1999) Pre- and postsynaptic distribution of cannabinoid and mu opioid receptors in rat spinal cord. Brain Res 822:17-25.

Hubscher CH, Berkley KJ (1995) Spinal and vagal influences on the responses of rat solitary nucleus neurons to stimulation of uterus, cervix and vagina. Brain Res 702:251-254.

Ibuki R, Haga N, Muramatsu S, Mizumoto A, Itoh Z (1997) Long-term observations of uterine contractions in nonpregnant dogs. Biol Reprod 56:632-639.

Ishac EJ, Jiang L, Lake KD, Varga K, Abood ME, Kunos G (1996) Inhibition of exocytotic noradrenaline release by presynaptic cannabinoid CB1 receptors on peripheral sympathetic nerves. $\mathrm{Br} \mathrm{J}$ Pharmacol 118:2023-2028.

Izzo AA, Fezza F, Capasso R, Bisogno T, Pinto L, Iuvone T, Esposito G, Mascolo N, DiMarzo V, Capasso F (2001) Cannabinoid CB1-receptor mediated regulation of gastrointestinal motility in mice in a model of intestinal inflammation. Br J Pharmacol 134:563-570.

Jaggar SI, Hasnie FS, Sellaturay S, Rice AS (1998a) The antihyperalgesic actions of the cannabinoid anandamide and the putative CB2 receptor agonist palmitoylethanolamide in visceral and somatic inflammatory pain. Pain 76:189-199.

Jaggar SI, Sellaturay S, Rice AS (1998b) The endogenous cannabinoid anandamide, but not the CB2 ligand palmitoylethanolamide, prevents the viscero-visceral hyper-reflexia associated with inflammation of the rat urinary bladder. Neurosci Lett 253:123-126.

Jonsson KO, Vandevoorde S, Lambert DM, Tiger G, Fowler CJ (2001) Effects of homologues and analogues of palmitoylethanolamide upon the inactivation of the endocannabinoid anandamide. $\mathrm{Br} \mathrm{J}$ Pharmacol 133:1263-1275.

Jordan VC, Castracane VD (1976) The effect of reported prostaglandin synthetase inhibitors on estradiol-stimulated uterine prostaglandin biosynthesis in vivo in the ovariectomized rat. Prostaglandins 12:1073-1081.

Joy JE, Watson Jr SJ, Benson Jr JA (1999) Marijuana and Medicine. Assessing the Science Base. Washington DC: National Academy.

Krall JF, Barrett JD, Jamgotchian N, Korenman SG (1984) Interaction of prostaglandin E2 and beta-adrenergic catecholamines in the regulation of uterine smooth muscle motility and adenylate cyclase in the rat. J Endocrinol 102:329-336.

Malinowska B, Godlewski G, Bucher B, Schlicker E (1997) Cannabinoid $\mathrm{CB} 1$ receptor-mediated inhibition of the neurogenic vasopressor response in the pithed rat. Naunyn Schmiedebergs Arch Pharmacol 356:197-202.

Malinowska B, Piszcz J, Koneczny B, Hryniewicz A, Schlicker E (2001) Modulation of the cardiac autonomic transmission of pithed rats by presynaptic opioid OP4 and cannabinoid CB1 receptors. Naunyn Schmiedebergs Arch Pharmacol 364:233-241.

Martin RS, Luong LA, Welsh NJ, Eglen RM, Martin GR, MacLennan SJ (2000) Effects of cannabinoid receptor agonists on neuronally-evoked contractions of urinary bladder tissues isolated from rat, mouse, pig, dog, monkey and human. Br J Pharmacol 129:1707-1715.

Martyn CN, Illis LS, Thom J (1995) Nabilone in the treatment of multiple sclerosis. Lancet 345:579.

Matsuda LA, Bonner TI, Lolait SJ (1993) Localization of cannabinoid receptor mRNA in rat brain. J Comp Neurol 327:535-550.

McMahon SB, Abel C (1987) A model for the study of visceral pain 
states: chronic inflammation of the chronic decerebrate rat urinary bladder by irritant chemicals. Pain 28:109-127.

Monga M, Weisbrodt NW, Andres RL, Sanborn BM (1993) The acute effect of cocaine exposure on pregnant human myometrial contractile activity. Am J Obstet Gynecol 169:782-785.

Moss NG, Harrington WW, Tucker MS (1997) Pressure, volume, and chemosensitivity in afferent innervation of urinary bladder in rats. Am J Physiol 272:R695-R703.

Niederhoffer N, Szabo B (1999) Effect of the cannabinoid receptor agonist W IN55212-2 on sympathetic cardiovascular regulation. Br J Pharmacol 126:457-466.

Niederhoffer N, Hansen HH, Fernandez-Ruiz JJ, Szabo B (2001) Effects of cannabinoids on adrenaline release from adrenal medullary cells. Br J Pharmacol 134:1319-1327.

O'Shaughnessy WB (1842) On the preparations of the Indian hemp, or gunja (Cannabis Indica). Trans Med Phys Soc 8:421-469.

Okamura T, Ghoneim HA, Ayajiki K, Ammar ES, Toda N (2000) Mechanisms underlying contraction and relaxation induced by nerve stimulation in monkey uterine arteries. Pharmacology 61:251-256.

Palazzo E, Marabese I, de Novellis V, Oliva P, Rossi F, Berrino L, Rossi F, Maione S (2001) Metabotropic and NMDA glutamate receptors participate in the cannabinoid-induced antinociception. Neuropharmacology 40:319-326.

Papka RE, Thompson BD, Schmidt HH (1996) Identification of uterinerelated sympathetic neurons in the rat inferior mesenteric ganglion: neurotransmitter content and afferent input. J Auton Nerv Syst 59:51-59.

Paria BC, Dey SK (2000) Ligand-receptor signaling with endocannabinoids in preimplantation embryo development and implantation. Chem Phys Lipids 108:211-220.

Pertwee RG (1999) Pharmacology of cannabinoid receptor ligands. Curr Med Chem 6:635-664.

Pertwee RG, Fernando SR (1996) Evidence for the presence of cannabinoid $\mathrm{CB} 1$ receptors in mouse urinary bladder. $\mathrm{Br} \mathrm{J}$ Pharmacol 118:2053-2058

Pettit DA, Harrison MP, Olson JM, Spencer RF, Cabral GA (1998)
Immunohistochemical localization of the neural cannabinoid receptor in rat brain. J Neurosci Res 51:391-402.

Richardson JD, Kilo S, Hargreaves KM (1998) Cannabinoids reduce hyperalgesia and inflammation via interaction with peripheral CB1 receptors. Pain 75:111-119.

Sant GR, Theoharides TC (1994) The role of the mast cell in interstitial cystitis. Urol Clin North Am 21:41-53.

Sato S, Hayashi RH, Garfield RE (1989) Mechanical responses of the rat uterus, cervix, and bladder to stimulation of hypogastric and pelvic nerves in vivo. Biol Reprod 40:209-219.

Sato Y, Hotta H, Nakayama H, Suzuki H (1996) Sympathetic and parasympathetic regulation of the uterine blood flow and contraction in the rat. J Auton Nerv Syst 59:151-158.

Schlicker E, Göthert M (1998) Interactions between the presynaptic alpha2-autoreceptor and presynaptic inhibitory heteroreceptors on noradrenergic neurones. Brain Res Bull 47:129-132.

Schlicker E, Timm J, Zentner J, Göthert M (1997) Cannabinoid CB1 receptor-mediated inhibition of noradrenaline release in the human and guinea-pig hippocampus. Naunyn Schmiedebergs Arch Pharmacol 356:583-589.

Schmid PC, Paria BC, Krebsbach RJ, Schmid HH, Dey SK (1997) Changes in anandamide levels in mouse uterus are associated with uterine receptivity for embryo implantation. Proc Natl Acad Sci USA 94:4188-4192.

Showalter VM, Compton DR, Martin BR, Abood ME (1996) Evaluation of binding in a transfected cell line expressing a peripheral cannabinoid receptor (CB2): identification of cannabinoid receptor subtype selective ligands. J Pharmacol Exp Ther 278:989-999.

Temple JL, Bradshaw HB, Wood E, Berkley KJ (1999) Effects of hypogastric neurectomy on escape responses to uterine distention in the rat Pain [Suppl] 6:S13-S20.

Vera PL, Nadelhaft I (1992) Afferent and sympathetic innervation of the dome and the base of the urinary bladder of the female rat. Brain Res Bull 29:651-658.

Wang FL, Dombrowski MP, Hurd WW (1996) Cocaine and betaadrenergic receptor function in pregnant myometrium. Am J Obstet Gynecol 175:1651-1653. 